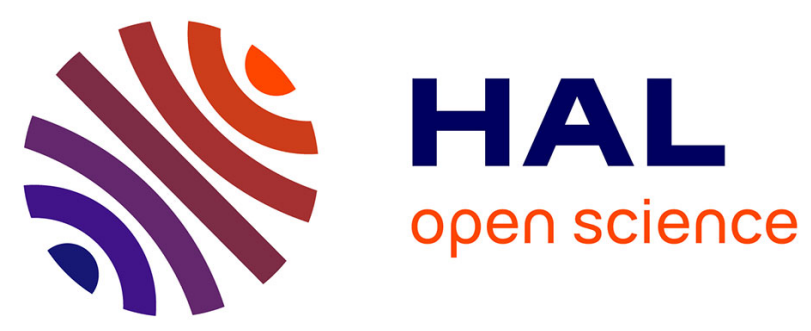

\title{
Electric and magnetic dipole strength in ${ }^{54} \mathbf{F e}$
}

R. Schwengner, R. Massarczyk, R. Beyer, M. Bhike, B. A. Brown, S.

Krishichayan, K. Sieja, W. Tornow, D. Bemmerer, Maik Butterling, et al.

\section{To cite this version:}

R. Schwengner, R. Massarczyk, R. Beyer, M. Bhike, B. A. Brown, et al.. Electric and magnetic dipole strength in ${ }^{54}$ Fe. Physical Review C, 2020, 101, pp.064303. 10.1103/PhysRevC.101.064303 . hal-02961162

\section{HAL Id: hal-02961162 \\ https://hal.science/hal-02961162}

Submitted on 8 Oct 2020

HAL is a multi-disciplinary open access archive for the deposit and dissemination of scientific research documents, whether they are published or not. The documents may come from teaching and research institutions in France or abroad, or from public or private research centers.
L'archive ouverte pluridisciplinaire HAL, est destinée au dépôt et à la diffusion de documents scientifiques de niveau recherche, publiés ou non, émanant des établissements d'enseignement et de recherche français ou étrangers, des laboratoires publics ou privés. 


\title{
Electric and magnetic dipole strength in ${ }^{54} \mathrm{Fe}$
}

\author{
R. Schwengner ${ }^{1},{ }^{1}$ R. Massarczyk, ${ }^{2}$ R. Beyer, ${ }^{1}$ M. Bhike, ${ }^{3,4}$ B. A. Brown, ${ }^{5}$ Krishichayan, ${ }^{3,4}$ K. Sieja, ${ }^{6,7}$ W. Tornow, ${ }^{3,4}$ \\ D. Bemmerer, ${ }^{1}$ M. Butterling, ${ }^{1}$ V. Derya,${ }^{8}$ M. Dietz, ${ }^{1}$ F. Fiedler, ${ }^{1}$ U. Friman-Gayer, ${ }^{9,}{ }^{*}$ A. Frotscher, ${ }^{1, \dagger}$ M. Grieger, ${ }^{1}$ \\ A. Hartmann, ${ }^{1}$ A. R. Junghans, ${ }^{1}$ T. Kögler, ${ }^{1,10}$ F. Ludwig, ${ }^{1}$ B. Lutz, ${ }^{1}$ H. Pai, ${ }^{11}$ T. Szücs, ${ }^{1}$ M. P. Takács, ${ }^{1, \ddagger}$ and A. Wagner ${ }^{1}$ \\ ${ }^{1}$ Helmholtz-Zentrum Dresden-Rossendorf, 01328 Dresden, Germany \\ ${ }^{2}$ Los Alamos National Laboratory, Los Alamos, New Mexico 87545, USA \\ ${ }^{3}$ Department of Physics, Duke University, Durham, North Carolina 27708, USA \\ ${ }^{4}$ Triangle Universities Nuclear Laboratory, Durham, North Carolina 27708, USA \\ ${ }^{5}$ National Superconducting Cyclotron Laboratory and Department of Physics and Astronomy, \\ Michigan State University, East Lansing, Michigan 48824, USA \\ ${ }^{6}$ Université de Strasbourg, IPHC, 67037 Strasbourg, France \\ ${ }^{7}$ CNRS, UMR7178, 67037 Strasbourg, France \\ ${ }^{8}$ Institut für Kernphysik, Universität zu Köln, 50937 Köln, Germany \\ ${ }^{9}$ Institut für Kernphysik, Technische Universität Darmstadt, 64289 Darmstadt, Germany \\ ${ }^{10}$ OncoRay-National Center for Radiation Research in Oncology, Faculty of Medicine and University Hospital Carl Gustav Carus, \\ Technische Universität Dresden, 01062 Dresden, Germany \\ ${ }^{11}$ Nuclear Physics Division, Saha Institute of Nuclear Physics, Kolkata-700064, India
}

(Received 4 March 2020; revised manuscript received 27 April 2020; accepted 22 May 2020; published 8 June 2020)

\begin{abstract}
The dipole strength of the $N=28$ closed-shell nuclide ${ }^{54} \mathrm{Fe}$ was studied in photon-scattering experiments using bremsstrahlung produced with electron beams of kinetic energies of 7.5 and $13.9 \mathrm{MeV}$ at the $\gamma$ ELBE facility as well as using quasimonoenergetic and linearly polarized photon beams of 26 different energies within the range from 5.5 to $11.4 \mathrm{MeV}$ at the $\mathrm{HI} \gamma \mathrm{S}$ facility. About $100 \mathrm{~J}=1$ states were newly identified, out of them 19 with $1^{+}$and 30 with $1^{-}$assignments. The quasicontinuum of unresolved transitions was included in the analysis of the spectra and the intensities of branching transitions were estimated on the basis of simulations of statistical $\gamma$-ray cascades. As a result, the photoabsorption cross section up to the neutron-separation energy was determined and compared with predictions of the statistical reaction model. The experimental $M 1$ strengths from resolved $1^{+}$states are compared with results of large-scale shell-model calculations.
\end{abstract}

DOI: 10.1103/PhysRevC.101.064303

\section{INTRODUCTION}

The experimental and theoretical descriptions of photonuclear reactions and the inverse radiative-capture reactions are crucial for the understanding of particular astrophysical processes of element synthesis. An important ingredient to the calculations of reaction cross sections within the statistical reaction model is photon strength functions, which describe average transition strengths in the energy region of the quasicontinuum of nuclear states at high excitation energies. The standard electric dipole strength functions used in statistical

\footnotetext{
*Present address: Department of Physics and Astronomy, University of North Carolina at Chapel Hill, Chapel Hill, North Carolina 27599, USA and Triangle Universities Nuclear Laboratory, Durham, North Carolina 27708, USA.

${ }^{\dagger}$ Present address: Institut für Kernphysik, Technische Universität Darmstadt, 64289 Darmstadt, Germany.

${ }^{\ddagger}$ Present address: Physikalisch-Technische Bundesanstalt, 38116 Braunschweig, Germany.
}

reaction-model calculations are Lorentz functions adjusted to $(\gamma, n)$ reaction data that represent the isovector giant dipole resonance (GDR) [1]. To test whether the low-energy tail of the Lorentz approximation describes the strength function below the neutron-separation energy $\left(S_{n}\right)$ correctly, experiments using other reactions are needed, in which nuclear levels below the particle-separation energies are excited [2].

A specific reaction for the study of dipole excitations up to the particle thresholds is photon scattering $\left(\gamma, \gamma^{\prime}\right)$, also called nuclear resonance fluorescence (NRF), in which the angular momentum $L=1$ is predominantly transferred by the incident photons and states with spins and parities of $J^{\pi}=1^{+}$and $1^{-}$are excited from the ground state in an eveneven nucleus. Energy-integrated scattering cross sections of the excited states are determined from the intensities of the respective $\gamma$ transitions back to the ground state and are used to determine reduced transition strengths for electric dipole $(E 1)$ and magnetic dipole $(M 1)$ transitions.

In recent photon-scattering studies using broad-band bremsstrahlung at the $\gamma$ ELBE facility [3] of the HelmholtzZentrum Dresden-Rossendorf (HZDR), Germany, as well as 
using quasimonoenergetic, polarized photon beams produced at the high-intensity $\gamma$-ray source $(\mathrm{HI} \gamma \mathrm{S})$ [4], operated by the Triangle Universities Nuclear Laboratory (TUNL) in Durham, North Carolina, USA, we observed enhanced strength on top of the low-energy tail of the GDR, for example, in $N=50$ isotones [5], in Mo isotopes with $N=50$ to 58 [6], and in Xe isotopes with $N=70$ to $N=80$ [7]. Such enhanced $E 1$ strength in the excitation-energy region from about 6 to $10 \mathrm{MeV}$ is considered as a pygmy dipole resonance (PDR) [8-10]. To investigate the possible influence of the PDR on cross sections and reaction rates, the experimental strength distributions were used as an input for statistical reactionmodel calculations. As an example, the photoabsorption cross section deduced from $(\gamma, n)$ data obtained at $\mathrm{HI} \gamma \mathrm{S}$ and the one from $\left(\gamma, \gamma^{\prime}\right)$ data obtained at $\gamma$ ELBE for ${ }^{86} \mathrm{Kr}$ were combined and used as an input for the calculation of $(n, \gamma)$ reaction rates of the $s$-process branching-point nuclide ${ }^{85} \mathrm{Kr}$, which provided novel information about stellar processes [11]. The experiments performed on Xe isotopes at $\mathrm{HI} \gamma \mathrm{S}$ and at $\gamma \mathrm{ELBE}$ revealed that the neutron excess causes the dominating effect on the development of $E 1$ strength in the pygmy region, whereas the deformation plays a minor role only [7]. The experiments at $\mathrm{HI} \gamma \mathrm{S}$ using polarized photon beams allow a distinction between the $E 1$ and $M 1$ contributions to the photoabsorption cross sections. An investigation of ${ }^{128} \mathrm{Xe}$ and ${ }^{134} \mathrm{Xe}$ including strength in the quasicontinuum of unresolved states proved that the main part $(\approx 90 \%)$ of the photoabsorption cross section in the pygmy region is of $E 1$ character, while the $M 1$ cross section gives a comparably small contribution only [12]. This relation seems to change when going to lighter nuclides, such as the ones in the iron-nickel region. In ${ }^{50} \mathrm{Cr}[13,14],{ }^{52} \mathrm{Cr}[15,16],{ }^{54} \mathrm{Cr}[14],{ }^{56} \mathrm{Fe}$ [17], and ${ }^{58,60} \mathrm{Ni}$ [18], several strong isolated $M 1$ excitations have been observed. A detailed investigation of these finding requires the study of further nuclei with varing properties, such as nuclides at shell closures and within open shells. This may serve as a test of current phenomenological approximations of the $E 1$ and $M 1$ strength functions used as an input for statistical reaction-model calculations.

To address these issues, we initiated a study of the nuclide ${ }^{54} \mathrm{Fe}$ having a closed neutron shell $(N=28)$. In a previous NRF experiment, one $J=1$ state at $6129 \mathrm{keV}$ with a level width of $\Gamma=27(4) \mathrm{meV}$ was identified without parity assignment [19]. We performed experiments at $\gamma$ ELBE at electron energies of 7.5 and $13.9 \mathrm{MeV}$ to determine integrated scattering cross sections of excited states and the photoabsorption cross section including contributions from the quasicontinuum of states. Furthermore, experiments at $\mathrm{HI} \gamma \mathrm{S}$ using photon-beam energies between 5.5 and $11.4 \mathrm{MeV}$ were conducted to identify individual $M 1$ and $E 1$ transitions in ${ }^{54} \mathrm{Fe}$.

\section{EXPERIMENTAL METHODS AND RESULTS}

\section{A. The photon-scattering method}

In photon-scattering experiments, the energy- and solidangle-integrated scattering cross section $I_{S}$ of an excited state at the energy $E_{x}$ is deduced from the measured intensity of the respective transition to the ground state. It can be determined relative to known integrated scattering cross sections. In the present experiments, we used the integrated scattering cross sections $I_{s}\left(E_{x}^{\mathrm{B}}\right)$ of states in ${ }^{11} \mathrm{~B}[20]$ and their angular correlations including mixing ratios $[21]$ as a reference:

$$
\begin{aligned}
\frac{I_{s}\left(E_{x}\right)}{I_{s}\left(E_{x}^{\mathrm{B}}\right)}= & \left(\frac{I_{\gamma}\left(E_{\gamma}, \theta\right)}{W\left(E_{\gamma}, \theta\right) \Phi_{\gamma}\left(E_{x}\right) N_{N}}\right) \\
& \times\left(\frac{I_{\gamma}\left(E_{\gamma}^{\mathrm{B}}, \theta\right)}{W\left(E_{\gamma}^{\mathrm{B}}, \theta\right) \Phi_{\gamma}\left(E_{x}^{\mathrm{B}}\right) N_{N}^{\mathrm{B}}}\right)^{-1} .
\end{aligned}
$$

Here, $I_{\gamma}\left(E_{\gamma}, \theta\right)$ and $I_{\gamma}\left(E_{\gamma}^{\mathrm{B}}, \theta\right)$ denote the detectorefficiency-corrected measured intensities of a considered ground-state transition at $E_{\gamma}$ and of a ground-state transition in ${ }^{11} \mathrm{~B}$ at $E_{\gamma}^{\mathrm{B}}$, respectively, observed at an angle $\theta$ to the beam. $W\left(E_{\gamma}, \theta\right)$ and $W\left(E_{\gamma}^{\mathrm{B}}, \theta\right)$ describe the angular correlations of these transitions. The quantities $N_{N}$ and $N_{N}^{\mathrm{B}}$ are the numbers of nuclei in the ${ }^{54} \mathrm{Fe}$ and ${ }^{11} \mathrm{~B}$ targets, and $\Phi_{\gamma}\left(E_{x}\right)$ and $\Phi_{\gamma}\left(E_{x}^{\mathrm{B}}\right)$ stand for the photon fluxes at the energy of the considered level and at the energy of a level in ${ }^{11} \mathrm{~B}$, respectively.

The integrated scattering cross section is related to the partial width of the ground-state transition $\Gamma_{0}$ according to

$$
I_{s}=\int \sigma_{\gamma \gamma} d E=\left(\frac{\pi \hbar c}{E_{x}}\right)^{2} \frac{2 J_{x}+1}{2 J_{0}+1} \frac{\Gamma_{0}^{2}}{\Gamma},
$$

where $\sigma_{\gamma \gamma}$ is the elastic-scattering cross section; $E_{x}, J_{x}$, and $\Gamma$ denote energy, spin, and total width of the excited level, respectively; and $J_{0}$ is the spin of the ground state.

The determination of the level widths is complicated by two problems. First, a considered level can be fed by transitions from higher-lying states, and second, such a level can deexcite to low-lying excited states (inelastic scattering) in addition to the deexcitation to the ground state (elastic scattering). In the case of feeding, the measured intensity of the ground-state transition is greater than the one resulting from a direct excitation only. As a consequence, the integrated scattering cross section $I_{s+f}$ deduced from this intensity contains a portion, $I_{f}$, originating from feeding in addition to the true integrated scattering cross section $I_{s}$. In the case of inelastic scattering, inelastic and subsequent cascade transitions appear in the measured spectrum in addition to ground-state transitions. To deduce the partial width of the ground-state transition $\Gamma_{0}$ and the absorption cross section, the knowledge of the branching ratio $b_{0}=\Gamma_{0} / \Gamma$ is needed.

Spins of excited states can be deduced by comparing experimental ratios of $\gamma$-ray intensities, measured at two angles, with theoretical predictions. The optimum combination includes angles of $90^{\circ}$ and $127^{\circ}$ because the respective ratios for the spin sequences $0-$ $1-0$ and $0-2-0$ differ most at these angles. The expected values are $W\left(90^{\circ}\right) / W\left(127^{\circ}\right)_{0-1-0}=0.74$ and $W\left(90^{\circ}\right) / W\left(127^{\circ}\right)_{0-2-0}=2.15$ taking into account opening angles of $16^{\circ}$ and $14^{\circ}$ of the detectors placed at $90^{\circ}$ and $127^{\circ}$, respectively, in the setup at $\gamma \mathrm{ELBE}$.

Parities of excited states can be determined by measuring the linear polarizations of the ground-state transitions. These are deduced from experimental asymmetries of intensities 
measured parallel or perpendicular to the polarization plane of the incident polarized photon beam at $\mathrm{HI} \gamma \mathrm{S}$.

\section{B. The target}

The target consisted of $1.4984 \mathrm{~g}$ of iron, enriched to $99.93 \%$ in ${ }^{54} \mathrm{Fe}$ and shaped to a disk of $20 \mathrm{~mm}$ in diameter. In the experiments at $\gamma$ ELBE, the target was combined with $0.300 \mathrm{~g}$ of boron, enriched to $99.5 \%$ in ${ }^{11} \mathrm{~B}$ and also formed to a disk of $20 \mathrm{~mm}$ in diameter. The known integrated scattering cross sections of states in ${ }^{11} \mathrm{~B}$ were used to determine the photon flux.

\section{Detector response}

For the determination of the integrated scattering cross sections according to Eq. (1), the efficiencies of the detectors and the photon flux are needed. The determination of the absorption cross section described in Sec. III requires in addition a correction of the experimental spectra for atomic processes induced by the impinging photons in the target material and for ambient background radiation. The detector response was simulated using the program package GEANT4 [22-24]. The reliability of the simulations was tested by comparing simulated spectra with measured ones as described, for example, in Refs. [25-28].

The absolute efficiencies of the high-purity germanium (HPGe) detectors in the setup at $\gamma$ ELBE were determined experimentally up to $2.4 \mathrm{MeV}$ from measurements with a ${ }^{226} \mathrm{Ra}$ calibration source. For interpolation, an efficiency curve calculated with GEANT4 and scaled to the absolute experimental values was used. A check of the simulated efficiency curve up to about $9 \mathrm{MeV}$ was performed via various $(p, \gamma)$ reactions at the HZDR Tandetron accelerator. The efficiency values deduced from these measurements agree with the simulated values within their uncertainties [29]. Similar results were obtained for the resonances at 4.44 and $11.66 \mathrm{MeV}$ in ${ }^{12} \mathrm{C}$ populated in the ${ }^{11} \mathrm{~B}(p, \gamma)$ reaction at the TUNL van-deGraaff accelerator [30].

\section{Experiments with bremsstrahlung at $\gamma$ ELBE}

The nuclide ${ }^{54} \mathrm{Fe}$ was studied in two experiments at $\gamma$ ELBE [3]. Bremsstrahlung was produced using electron beams of 7.5 and $13.9 \mathrm{MeV}$ kinetic energy. In the measurement at $7.5 \mathrm{MeV}$, the electron beam hit a niobium foil of $5 \mu \mathrm{m}$ in thickness acting as a radiator at an average current of about $650 \mu \mathrm{A}$. In the measurement at $13.9 \mathrm{MeV}$, the radiator had a thickness of $12.5 \mu \mathrm{m}$ and the average current was about $530 \mu \mathrm{A}$. A 10-cm-thick aluminum absorber (beam hardener) was placed behind the radiator to reduce the low-energy intensity of the bremsstrahlung spectrum in the measurement at $13.9 \mathrm{MeV}$. The photon beam, collimated by a $260-\mathrm{cm}-\mathrm{long}$ pure-aluminum collimator with a conical borehole of $8 \mathrm{~mm}$ in diameter at the entrance and $24 \mathrm{~mm}$ in diameter at the exit, impinged onto the target, placed $200 \mathrm{~cm}$ behind the collimator exit, with a flux of about $10^{9} \mathrm{~s}^{-1}$ in a spot of $38 \mathrm{~mm}$ in diameter. Scattered photons were measured with four HPGe detectors that have an efficiency of $100 \%$ relative to an $\mathrm{NaI}$ detector of $7.6 \mathrm{~cm}$ in diameter and $7.6 \mathrm{~cm}$ in length.

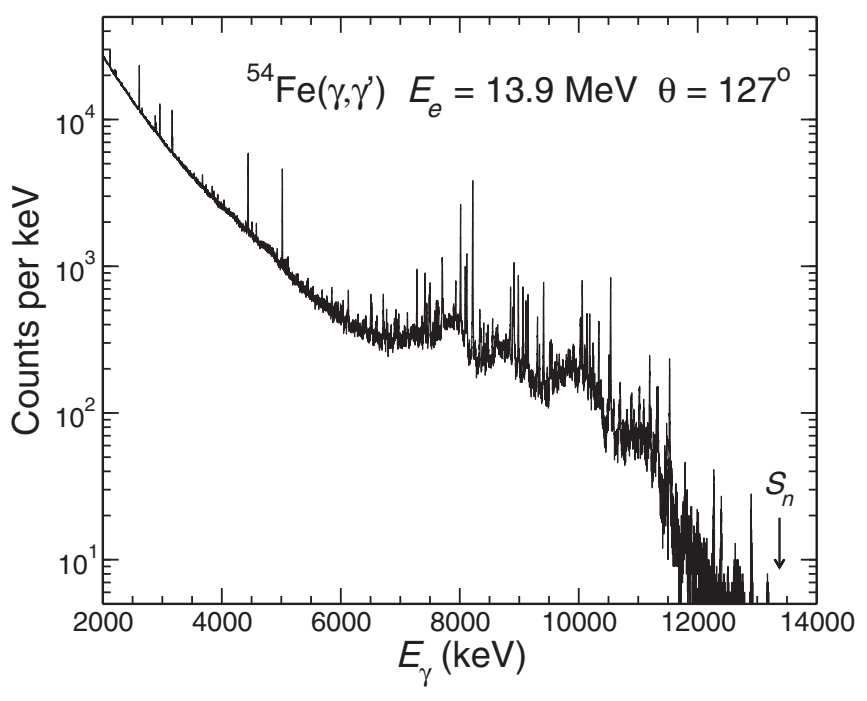

FIG. 1. Part of a spectrum of photons scattered from ${ }^{54} \mathrm{Fe}$ combined with ${ }^{11} \mathrm{~B}$, measured during the irradiation with bremsstrahlung produced by electrons of an energy of $E_{e}^{\mathrm{kin}}=13.9 \mathrm{MeV}$. This spectrum is the sum of the spectra measured with the two detectors placed at $127^{\circ}$ relative to the beam.

All HPGe detectors were surrounded by escape-suppression shields made of bismuth germanate scintillation detectors of $3 \mathrm{~cm}$ in thickness. Two HPGe detectors were placed vertically at $127^{\circ}$ relative to the photon-beam direction and a distance of $32 \mathrm{~cm}$ from the target. The other two HPGe detectors were positioned in a horizontal plane at $90^{\circ}$ to the beam and a distance of $28 \mathrm{~cm}$ from the target. Absorbers of $8 \mathrm{~mm}$ lead plus $3 \mathrm{~mm}$ copper were placed in front of the detectors at $90^{\circ}$ and of $3 \mathrm{~mm}$ lead plus $3 \mathrm{~mm}$ copper in front of the detectors at $127^{\circ}$. Spectra of scattered photons were measured for 86 and $141 \mathrm{~h}$ in the experiments at 7.5 and $13.9 \mathrm{MeV}$ electron energy, respectively. Part of the spectrum including events measured with the two detectors placed at $127^{\circ}$ relative to the beam at an electron energy of $13.9 \mathrm{MeV}$ is shown in Fig. 1.

The absolute photon fluxes in the two measurements at $\gamma$ ELBE were determined from intensities and known integrated scattering cross sections of transitions in ${ }^{11} \mathrm{~B}$. For interpolation, the photon flux was calculated using a bremsstrahlung computer code [31] based on the Born approximation with Coulomb correction [32] and including an atomic screening correction [33]. In addition, the flux was corrected for the attenuation by the beam hardener. The calculated flux curves were scaled to the experimental values obtained at the energies of levels in ${ }^{11} \mathrm{~B}$. The experimental flux values and the calculated curves are shown in Fig. 2.

The measurements at two electron energies allowed us to identify inelastic transitions from high-lying levels that feed low-lying levels. Transitions found in the measurement at $E_{e}^{\mathrm{kin}}=7.5 \mathrm{MeV}$ are assumed to be ground-state transitions. Transitions additionally observed up to $7.5 \mathrm{MeV}$ in the measurement at $13.9 \mathrm{MeV}$ are considered as inelastic transitions from high-lying to low-lying excited states. By comparing the respective spectra, these inelastic transitions were sorted out. The remaining ground-state transitions were used to derive the 


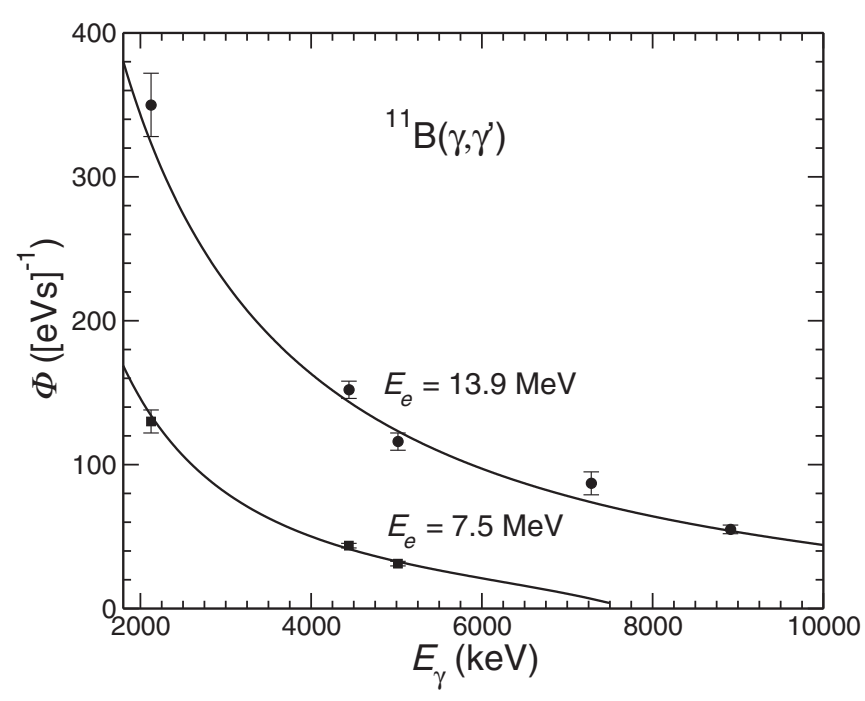

FIG. 2. Absolute photon flux on the target deduced from intensities of known transitions in ${ }^{11} \mathrm{~B}$ for the measurements at $E_{e}=$ 7.5 MeV (squares) and $E_{e}=13.9 \mathrm{MeV}$ (circles). The solid lines represent the calculated flux curves described in the text.

corresponding level energies that are listed in Table I together with spin assignments deduced from angular distributions of the ground-state transitions and with integrated scattering cross sections.

The detection limit for a $95 \%$ confidence level is defined as $A_{\mathrm{DL}}=2.8 \sqrt{2 B}$ [35], where $B$ is the integral over a background interval of full width at half maximum (FWHM) of a nearby peak, which has been applied in similar experiments [36]. The state with the smallest $I_{s}$ in Table I is the one at $5432 \mathrm{keV}$ with $I_{s}=1.9(3) \mathrm{eV}$ b. At this peak, one obtains $A_{\text {DL }} / A_{\text {PEAK }}=0.38(9)$ from the $127^{\circ}$ spectra at $\gamma$ ELBE, i.e., the $I_{s}$ of the $5432 \mathrm{keV}$ state is still more than twice the detection limit. Toward high energy, the background decreases rapidly, while FWHM increases. For the highest state given in Table I at $13171 \mathrm{keV}$, one obtains $A_{\mathrm{DL}} / A_{\mathrm{PEAK}}=0.33(9)$.

\section{E. Experiments with monoenergetic and linearly polarized photon beams at $\mathrm{HI} \gamma \mathrm{S}$}

Monoenergetic photon beams are produced at $\mathrm{HI} \gamma \mathrm{S}$ by Compton backscattering of a high-intensity free-electron laser (FEL) beam from an intense electron beam in the TUNL storage ring. Presently, the energy of the backward-scattered photons can be tuned in a wide energy range, from about 1 to $100 \mathrm{MeV}$, by changing the energy of the electron beam and the FEL wavelength [4]. The polarization of the FEL photons, defined by the magnetic field of the undulators, is mostly preserved during the Compton backscattering due to a negligible recoil effect, leading to the production of intense photon beams with a degree of polarization of nearly $100 \%$.

The measurements at $\mathrm{HI} \gamma \mathrm{S}$ were carried out at photonbeam energies of $5.5,5.7,5.9,6.1,6.3,6.6,6.8,7.0,7.2$, 7.6, 7.9, 8.1, 8.3, 8.5, 8.7, 8.9, 9.1, 9.3, 9.5, 9.8, 10.0, 10.3, $10.6,10.9,11.1$, and $11.4 \mathrm{MeV}$. The energy spread (FWHM) of the beam was about $3 \%$ of the energy using a $30.5-\mathrm{cm}$-long lead collimator with a cylindrical hole of $1.9 \mathrm{~cm}$ in diameter
TABLE I. Levels assigned to ${ }^{54} \mathrm{Fe}$.

\begin{tabular}{|c|c|c|c|c|}
\hline$E_{x}(\mathrm{keV})^{\mathrm{a}}$ & $I_{\gamma}\left(90^{\circ}\right) / I_{\gamma}\left(127^{\circ}\right)^{\mathrm{b}}$ & $A^{\mathrm{c}}$ & $J_{x}^{\pi \mathrm{d}}$ & $I_{S}(\mathrm{eV} \mathrm{b})^{\mathrm{e}}$ \\
\hline $1408.5(2)^{\mathrm{f}}$ & $1.19(10)$ & & & $18.3(20)^{\mathrm{g}}$ \\
\hline $2958.5(3)^{f}$ & $1.98(26)$ & & (2) & $8.1(11)^{\mathrm{g}}$ \\
\hline $3165.9(4)^{f}$ & $1.43(22)$ & & & $6.3(9)^{\mathrm{g}}$ \\
\hline $4581.7(7)^{\mathrm{f}}$ & $1.4(5)$ & & & $5.4(14)^{\mathrm{g}}$ \\
\hline $5085.0(6)$ & $0.72(23)$ & & 1 & $4.1(10)$ \\
\hline $5114.1(3)$ & $0.90(21)$ & & (1) & $4.7(7)$ \\
\hline $5431.8(7)$ & $1.0(3)$ & & (1) & $1.9(3)$ \\
\hline $6127.8(3)$ & $0.54(6)$ & $-0.87(7)$ & $1^{-}$ & $15.0(15)^{\mathrm{h}}$ \\
\hline $6522.7(3)$ & $0.73(12)$ & $-0.98(3)$ & $1^{-}$ & $10.1(13)$ \\
\hline $6787.6(9)$ & $0.71(26)$ & & 1 & $5.8(7)$ \\
\hline $6841.3(6)$ & $0.66(27)$ & $>0$ & $1^{+}$ & $6.5(17)$ \\
\hline $6914.2(9)$ & $0.78(17)$ & $>0$ & $1^{+}$ & $6.3(13)$ \\
\hline $6928.8(3)$ & $1.01(14)$ & & (1) & $19.4(23)$ \\
\hline $6946.5(6)$ & $1.3(3)$ & & & $9.6(18)$ \\
\hline $6975.1(4)$ & $0.55(15)$ & & 1 & $15.5(26)$ \\
\hline $7072.9(9)$ & $0.9(4)$ & & (1) & $5.6(14)$ \\
\hline $7085.4(7)$ & $0.7(3)$ & & 1 & $6.4(15)$ \\
\hline $7120.9(4)$ & $1.00(18)$ & $-0.74(18)$ & $(1)^{-}$ & $21.0(29)$ \\
\hline $7138.5(9)$ & & & & $7.4(17)$ \\
\hline $7334.8(7)$ & & & & $6.7(15)$ \\
\hline $7348.5(6)$ & $0.84(27)$ & & (1) & $8.6(16)$ \\
\hline $7367.3(4)$ & & & & $17.4(25)$ \\
\hline $7396.0(20)$ & & & & $5.6(27)$ \\
\hline $7415.4(3)$ & $1.07(7)$ & & & $79(8)$ \\
\hline 7447.2(3) & $0.94(12)$ & & (1) & $32(4)$ \\
\hline $7469.7(12)$ & & & & $9(3)$ \\
\hline 7487.4(4) & $1.06(11)$ & & & $42(5)$ \\
\hline 7553.3(9) & $0.7(3)$ & & 1 & $22(7)$ \\
\hline $7631.3(4)$ & & & & $33(4)$ \\
\hline $7645.8(5)$ & $1.66(18)$ & & & $23.4(28)$ \\
\hline $7723.2(5)$ & $1.25(12)$ & & & 31(3) \\
\hline 7898.2(5) & $1.07(17)$ & & & $19.7(28)$ \\
\hline 7916.5(9) & $1.6(4)$ & & & $9.8(20)$ \\
\hline 7933.8(2) & $1.09(9)$ & & & $55(5)$ \\
\hline $8014.8(2)$ & $0.74(3)$ & $-0.93(1)$ & $1^{-}$ & $344(3)$ \\
\hline $8093.2(2)$ & $0.71(5)$ & $-0.91(7)$ & $1^{-}$ & $106(10)$ \\
\hline $8119.2(2)$ & $0.73(4)$ & $+0.95(6)$ & $1^{+}$ & $148(13)$ \\
\hline $8163.7(7)$ & $0.49(28)$ & & 1 & $22(8)$ \\
\hline $8219.6(2)$ & $0.72(3)$ & $-0.91(2)$ & $1^{-}$ & $589(51)$ \\
\hline $8265.3(7)$ & $1.1(3)$ & & & $11.2(28)$ \\
\hline $8338.4(4)$ & $0.77(7)$ & $+0.92(4)$ & $1^{+}$ & $61(6)$ \\
\hline $8353.7(6)$ & $0.64(10)$ & $-0.98(7)$ & $1^{-}$ & $35(4)$ \\
\hline $8403.1(5)$ & $1.07(21)$ & & & $22(3)$ \\
\hline $8450.2(5)$ & $0.74(15)$ & $\approx-1$ & $1^{-}$ & $23(3)$ \\
\hline $8618.0(7)$ & $0.63(12)$ & $\approx+1$ & $1^{+}$ & $30(4)$ \\
\hline $8777.4(7)$ & $0.9(4)$ & & (1) & $18(5)$ \\
\hline $8841.0(5)$ & $1.06(17)$ & & & $18.8(26)$ \\
\hline $8855.5(2)$ & $0.69(4)$ & $+0.98(2)$ & $1^{+}$ & 102(9) \\
\hline 8897.1(3) & $0.77(5)$ & $-0.91(8)$ & $1^{-}$ & $67(6)$ \\
\hline
\end{tabular}


TABLE I. (Continued.)

\begin{tabular}{|c|c|c|c|c|}
\hline$E_{x}(\mathrm{keV})^{\mathrm{a}}$ & $I_{\gamma}\left(90^{\circ}\right) / I_{\gamma}\left(127^{\circ}\right)^{\mathrm{b}}$ & $A^{\mathrm{c}}$ & $J_{x}^{\pi \mathrm{d}}$ & $I_{s}(\mathrm{eV} \mathrm{b})^{\mathrm{e}}$ \\
\hline $8930.3(7)$ & $0.57(15)$ & & 1 & $17(3)$ \\
\hline $8986.0(2)$ & $0.68(4)$ & $+0.85(3)$ & $1^{+}$ & $142(13)$ \\
\hline $9065.2(3)$ & $0.63(4)$ & $+0.81(5)$ & $1^{+}$ & $130(12)$ \\
\hline 9113.1(2) & $0.73(5)$ & $+0.91(5)$ & $1^{+}$ & $94(8)$ \\
\hline $9128.9(7)$ & $1.22(22)$ & & & $13.8(23)$ \\
\hline $9149.3(2)$ & $0.66(4)$ & $+0.77(5)$ & $1^{+}$ & 104(9) \\
\hline $9307.3(3)$ & $0.62(6)$ & $>0$ & $1^{+}$ & $79(8)$ \\
\hline $9342.2(4)$ & $0.69(10)$ & $-0.98(4)$ & $1^{-}$ & $30(4)$ \\
\hline $9410.4(2)$ & $0.63(3)$ & $+0.85(5)$ & $1^{+}$ & $178(16)$ \\
\hline $9472.4(10)$ & & & & $48(18)$ \\
\hline $9515.4(5)$ & $0.54(11)$ & $\approx-1$ & $1^{-}$ & $49(7)$ \\
\hline $9538.0(6)$ & $0.41(8)$ & $\approx+1$ & $1^{+}$ & $47(7)$ \\
\hline $9908.6(6)$ & $0.93(17)$ & $>0$ & $\left(1^{+}\right)$ & $38(7)$ \\
\hline $9925.3(6)$ & $0.78(13)$ & $>0$ & $1^{+}$ & $38(5)$ \\
\hline $10035.5(3)$ & $0.63(6)$ & & & 93(9) \\
\hline $10060.5(2)$ & $0.62(3)$ & $>0$ & $1^{+}$ & $224(20)$ \\
\hline 10101.4(4) & $0.58(7)$ & $<0$ & $1^{-}$ & $52(6)$ \\
\hline 10119.2(5) & $0.93(12)$ & & (1) & $33(4)$ \\
\hline $10140.9(2)$ & $0.63(4)$ & $+0.93(2)$ & $1^{+}$ & $128(12)$ \\
\hline 10185.9(3) & $0.58(7)$ & $+0.93(2)$ & $1^{+}$ & $116(14)$ \\
\hline $10195.7(11)$ & $0.68(20)$ & & 1 & $33(9)$ \\
\hline $10224.2(11)$ & $0.42(23)$ & & 1 & $15(4)$ \\
\hline $10240.4(5)$ & $0.63(8)$ & $-0.63(17)$ & $1^{-}$ & $58(7)$ \\
\hline $10250.5(6)$ & $0.74(11)$ & $-0.82(24)$ & $1^{-}$ & $42(6)$ \\
\hline 10339.4(2) & $0.59(5)$ & $-0.99(2)$ & $1^{-}$ & $138(13)$ \\
\hline 10376.9(9) & $0.9(4)$ & $-0.8(4)$ & $(1)^{-}$ & $32(9)$ \\
\hline 10487.5(8) & $0.50(20)$ & $-0.53(21)$ & $1^{-}$ & $21(8)$ \\
\hline $10510.5(4)$ & $0.55(11)$ & $-0.94(8)$ & $1^{-}$ & 71(11) \\
\hline $10538.1(2)$ & $0.62(3)$ & $+0.86(13)$ & $1^{+}$ & $327(29)$ \\
\hline $10590.7(7)$ & $0.40(17)$ & $-0.93(12)$ & $1^{-}$ & $21(6)$ \\
\hline 10701.2(4) & $0.57(6)$ & $-0.93(5)$ & $1^{-}$ & $46(5)$ \\
\hline $10802.5(14)$ & $0.22(15)$ & & 1 & 26(9) \\
\hline $10820.4(10)$ & $0.34(15)$ & & 1 & $27(8)$ \\
\hline $10880.2(7)$ & $0.57(11)$ & $-0.98(28)$ & $1^{-}$ & $29(4)$ \\
\hline 10891.3(7) & $0.59(11)$ & $-0.99(11)$ & $1^{-}$ & $27(4)$ \\
\hline 10926.1(7) & $0.51(18)$ & $-0.89(7)$ & $1^{-}$ & $15(3)$ \\
\hline 11021.7(6) & $0.47(9)$ & $<0$ & $1^{-}$ & $105(17)$ \\
\hline 11094.8(6) & $0.54(13)$ & & 1 & $32(5)$ \\
\hline $11159.8(10)$ & $0.23(15)$ & & 1 & $15(3)$ \\
\hline 11194.0(3) & $0.55(4)$ & $-0.97(4)$ & $1^{-}$ & $99(9)$ \\
\hline $11210.6(5)$ & $0.47(7)$ & $-0.89(13)$ & $1^{-}$ & $36(4)$ \\
\hline $11314.0(6)$ & $0.65(8)$ & $-0.82(13)$ & $1^{-}$ & $46(6)$ \\
\hline $11333.5(4)$ & $0.55(5)$ & $-0.84(19)$ & $1^{-}$ & 74(8) \\
\hline 11447.4(4) & $0.72(14)$ & & 1 & $11.2(22)$ \\
\hline $11480.6(6)$ & $0.74(16)$ & $-0.89(14)$ & $1^{-}$ & $37(6)$ \\
\hline 11530.6(3) & $0.63(5)$ & $-0.79(8)$ & $1^{-}$ & $156(15)$ \\
\hline $11561.4(12)$ & $0.43(15)$ & & 1 & $17(4)$ \\
\hline 11761.1(14) & $1.0(4)$ & & & $6.8(19)$ \\
\hline $11790.5(5)$ & $0.73(12)$ & & 1 & $28(4)$ \\
\hline
\end{tabular}

TABLE I. (Continued.)

\begin{tabular}{lcccc}
\hline \hline$E_{x}(\mathrm{keV})^{\mathrm{a}}$ & $I_{\gamma}\left(90^{\circ}\right) / I_{\gamma}\left(127^{\circ}\right)^{\mathrm{b}}$ & $A^{\mathrm{c}}$ & $J_{x}^{\pi \mathrm{d}}$ & $I_{s}(\mathrm{eV} \mathrm{b})^{\mathrm{e}}$ \\
\hline $11821.7(10)$ & $0.8(3)$ & $(1)$ & $9.9(23)$ \\
$12273.2(5)$ & $0.60(10)$ & 1 & $27(3)$ \\
$12397.0(7)$ & $0.35(11)$ & 1 & $25(4)$ \\
$12902.5(8)$ & $0.33(13)$ & 1 & $29(5)$ \\
$13171.4(15)$ & $0.19(9)$ & 1 & $6.0(10)$ \\
\hline
\end{tabular}

${ }^{a}$ Excitation energy. The uncertainty of this and the other quantities in the table is given in parentheses in units of the last digit. This energy value was deduced from the $\gamma$-ray energy measured at $127^{\circ}$ including a recoil and Doppler-shift correction.

${ }^{b}$ Ratio of the intensities measured at angles of $90^{\circ}$ and $127^{\circ}$. The expected values for an elastic dipole transition (spin sequence $0-$ $1-0$ ) and for an elastic quadrupole transition (spin sequence $0-$ $2-0)$ are 0.74 and 2.15 , respectively.

${ }^{\mathrm{c}}$ Azimuthal asymmetry $A=\left(I_{\gamma \mathrm{H}}-I_{\gamma \mathrm{V}}\right) /\left(I_{\gamma \mathrm{H}}+I_{\gamma \mathrm{V}}\right)$ of the intensities $I_{\gamma \mathrm{H}}$ and $I_{\gamma \mathrm{V}}$ measured with the detectors placed in a horizontal and vertical plane, respectively. A negative asymmetry indicates $E 1$ radiation and a positive asymmetry indicates $M 1 / E 2$ radiation.

${ }^{\mathrm{d}}$ Spin and parity deduced from angular correlation and azimuthal asymmetry, respectively, of the ground-state transition.

${ }^{\mathrm{e}}$ Energy-integrated scattering cross section. Below an excitation energy of $6.5 \mathrm{MeV}$ the value was deduced from the measurement at 7.5 MeV electron energy, otherwise the value was deduced from the measurement at $13.9 \mathrm{MeV}$.

${ }^{\mathrm{f}}$ This state is assumed to be a known $2^{+}$state [34].

${ }^{g}$ Value probably affected by feeding intensities.

${ }^{\mathrm{h}}$ This value compares to $I_{s}=7.5(11) \mathrm{eV} \mathrm{b}$ deduced from the level width given in Ref. [19].

positioned $60 \mathrm{~m}$ downstream from the collision point of the electrons with the FEL photons. The measuring time was on average about $3 \mathrm{~h}$ for each selected energy. The photon beam impinged onto the target with a flux of about $5 \times 10^{6} \mathrm{~s}^{-1}$ for the lowest energies up to $3 \times 10^{7} \mathrm{~s}^{-1}$ for the highest energies. Scattered photons were measured with four HPGe detectors of $60 \%$ relative efficiency, placed at polar angles of $90^{\circ}$ to the beam, two in the vertical plane and two in the horizontal plane through the beam tube. The type of radiation ( $E 1$ or $M 1 / E 2)$ was deduced from a comparison of the intensities of the transitions measured at the different azimuthal angles [21,37]. In the present setup, $E 1$ radiation is detected preferentially in the vertical detectors and $M 1$ radiation in the horizontal detectors. As an example, spectra measured at a beam energy of $10.6 \mathrm{MeV}$ are shown in Fig. 3 together with the corresponding section of the spectrum from Fig. 1. The spectra allow a clear distinction between $E 1$ and $M 1$ radiation. Azimuthal asymmetries $A=\left(I_{\gamma \mathrm{H}}-I_{\gamma \mathrm{V}}\right) /\left(I_{\gamma \mathrm{H}}+I_{\gamma \mathrm{V}}\right)$ deduced from the intensities $I_{\gamma \mathrm{H}}$ measured in the horizontal detectors and $I_{\gamma \mathrm{V}}$ measured in the vertical detectors are given in Table I together with the resulting parities' assignments to the emitting states. Inelastic transitions, for example, from the states in the excited energy region to the $2_{1}^{+}$state, could not be clearly identified because of the low statistics in these short measurements.

The $6523-\mathrm{keV}$ peak is the smallest observed at $\mathrm{HI} \gamma \mathrm{S}$ with a definite asymmetry determination. In the spectrum of the vertical detectors at $6.6 \mathrm{MeV}$ beam energy, one obtains for 


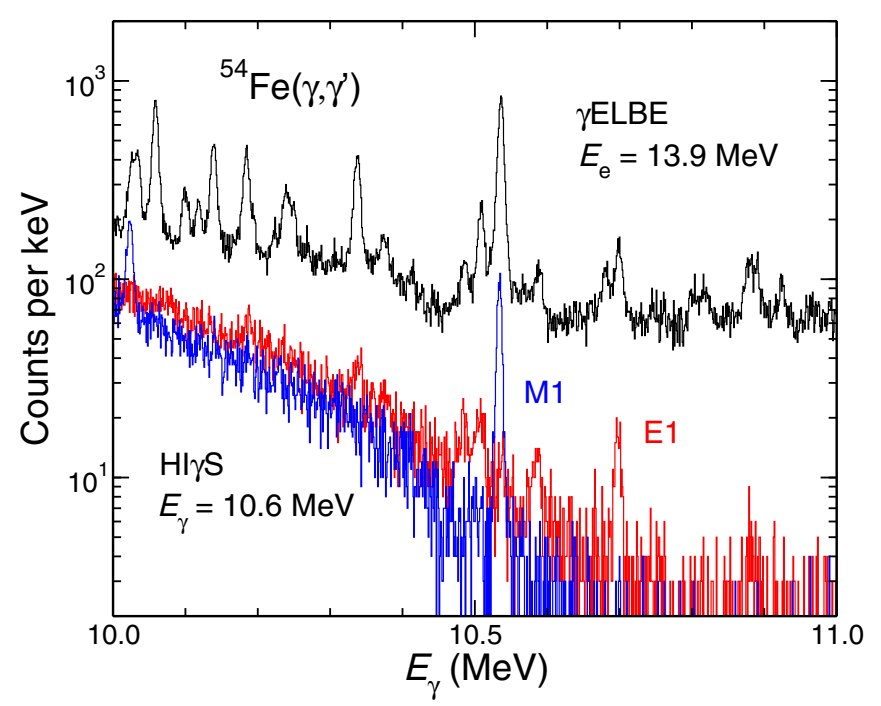

FIG. 3. Parts of spectra of photons scattered from ${ }^{54} \mathrm{Fe}$, measured during the irradiation with a quasimonoenergetic polarized photon beam of $10.6 \mathrm{MeV}$ at $\mathrm{HI} \gamma \mathrm{S}$. The spectrum plotted in red was measured with the vertical detectors and contains $E 1$ radiation, whereas the spectrum plotted in blue was measured with the horizontal detectors and contains $M 1$ radiation. For comparison, the corresponding section of the spectrum measured with bremsstrahlung of $E_{e}=13.9 \mathrm{MeV}$ at $\gamma \mathrm{ELBE}$ (cf. Fig. 1) is shown in black.

the detection limit (see Sec. IID) the relation $A_{\mathrm{DL}} / A_{\mathrm{PEAK}}=$ $0.30(4)$, compared with $A_{\mathrm{DL}} / A_{\mathrm{PEAK}}=0.12(1)$ at $\gamma \mathrm{ELBE}$.

\section{DETERMINATION OF THE DIPOLE-STRENGTH DISTRIBUTION}

The determination of the dipole-strength distribution and the related photoabsorption cross section requires the knowledge of the intensity distribution of the ground-state transitions and their branching ratios. As these cannot be derived directly from the measured spectra, we applied statistical methods to discriminate between $\gamma$ rays from nuclear excitations and photons scattered by atomic processes and to disentangle the intensity distributions of elastic and inelastic transitions in the quasicontinuum of nuclear levels.

First, a spectrum of the ambient background adjusted to the intensities of the transitions from ${ }^{40} \mathrm{~K}$ and ${ }^{208} \mathrm{Tl}$ decay in the in-beam spectrum was subtracted from the measured spectrum. To correct the spectrum for the detector response, spectra of monoenergetic $\gamma$ rays were calculated in steps of $10 \mathrm{keV}$ by using the simulation code GEANT4. Starting from the high-energy end of the experimental spectrum, the simulated spectra were subtracted sequentially (spectrum-stripping method) [38].

The background radiation produced by atomic processes in the ${ }^{54} \mathrm{Fe}$ target was obtained from a GEANT4 simulation. The simulated atomic background is compared with the responsecorrected spectrum in Fig. 4. As found in previous studies $[5,6,12,27,39-42]$ the continuum in the spectrum of $\gamma$ rays scattered from ${ }^{54} \mathrm{Fe}$ is considerably higher than the background due to atomic scattering. This continuum is formed by a large number of unresolved transitions with small intensities

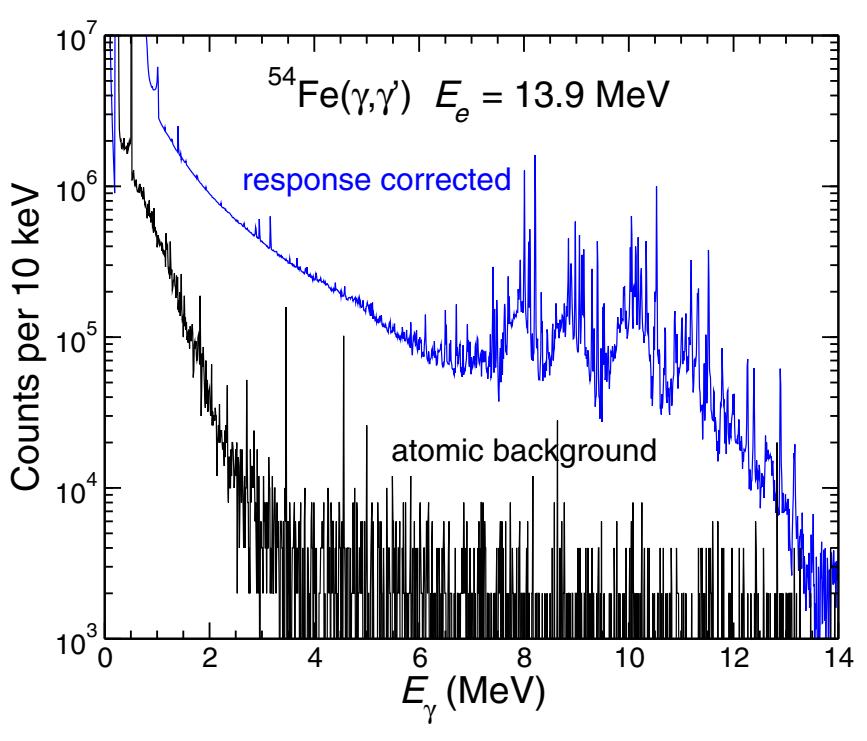

FIG. 4. Response-corrected spectrum of the two detectors placed at $127^{\circ}$ (blue) and simulated spectrum of photons scattered from the target to the detectors by atomic processes (black).

that are a consequence of the increasing nuclear level density at high excitation energies in connection with the finite detector resolution.

The intensity distribution resulting from the subtraction of the atomic background contains ground-state (elastic) transitions and, in addition, branching (inelastic) transitions to lower-lying excited states as well as transitions from those states to the ground state (cascade transitions). The different types of transitions cannot be clearly distinguished. However, for the determination of the photoabsorption cross section and the partial widths $\Gamma_{0}$, the intensities of the ground-state transitions are needed. Therefore, contributions of inelastic and cascade transitions have to be subtracted from the spectra. We corrected the intensity distributions by simulating $\gamma$-ray cascades from the levels in the whole energy range using the code $\gamma$ DEX [28,43]. This code works analogously to the strategy of the code DICEBOX [44] developed for $(n, \gamma)$ reactions, but in addition it includes also the excitation from the ground state. In the present simulations, level schemes (nuclear realizations) including states with $J=0, \ldots, 5$ were created. For the low-energy part of the level scheme below 3.5 $\mathrm{MeV}$, experimentally known levels were taken into account. Fluctuations of the partial widths were treated by applying the Porter-Thomas distribution [45]. Level densities were calculated by using the constant-temperature model [46] with the parameters $T=1.42(3) \mathrm{MeV}$ and $E_{0}=0.04(11) \mathrm{MeV}$ adjusted to experimental level densities [47]. In the individual nuclear realizations, the values of $T$ and $E_{0}$ were varied randomly within a Gaussian distribution with a $\sigma$ corresponding to the uncertainties given in Ref. [47]. The parity distribution of the level densities was modeled according to the information given in Ref. [48].

The first inputs for the photon strength function simulations were assumed to be Lorentz-shaped. For the $E 1$ strength a combination of three Lorentz functions (TLO), 


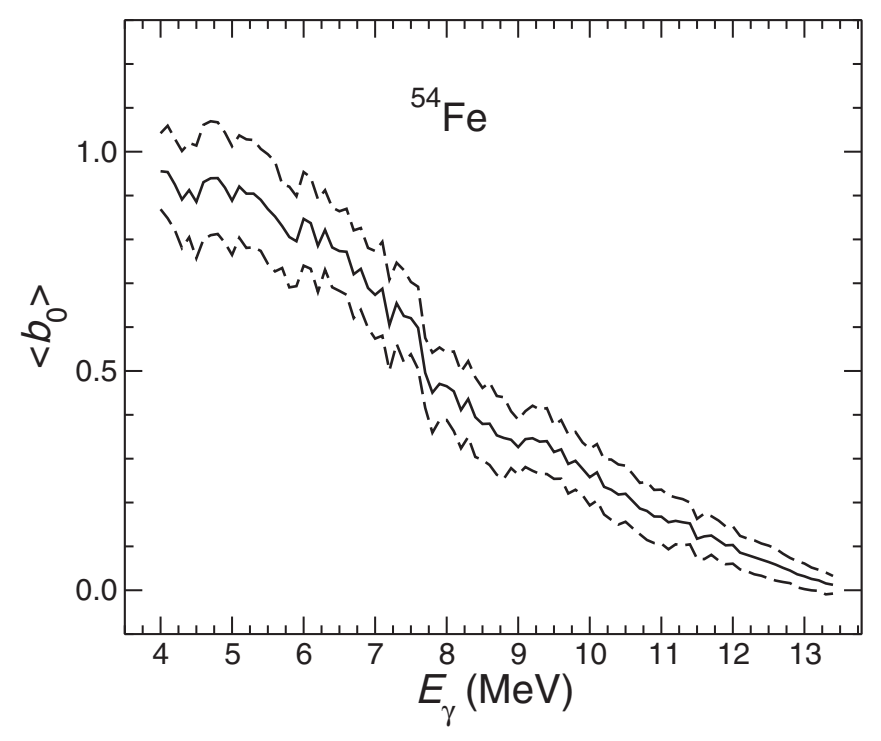

FIG. 5. Average branching ratios (solid line) with their uncertainty ranges (dashed lines) resulting from the simulations of statistical $\gamma$-ray cascades up to $S_{n}$ as described in the text.

with parameters as described in Refs. [49,50], was used with deformation parameters of $\beta_{2}=0.2$ [51] and $\gamma=30^{\circ}$ [52]. The parameters for the $M 1$ and $E 2$ strengths were taken from global parametrizations of $M 1$ spin-flip resonances and $E 2$ isoscalar resonances, respectively [53]. Spectra of $\gamma$-ray cascades were generated for groups of levels in 100-keV bins. Starting from the high-energy end of the experimental spectrum, which contains ground-state transitions only, the simulated intensities of the ground-state transitions were normalized to the experimental ones in the considered bin. The intensity distribution of the branching transitions was subtracted from the experimental spectrum. Applying this procedure step-by-step for each energy bin moving toward the low-energy end of the spectrum, one obtains the intensity distribution of the ground-state transitions. Simultaneously, the branching ratios $b_{0}(E)$ of the ground-state transitions are deduced for each energy bin $\Delta E$. In an individual nuclear realization, the branching ratio $b_{0}(E)$ is calculated as the ratio of the sum of the intensities of the ground-state transitions from all levels in $\Delta E$ to the total intensity of all transitions depopulating those levels to any low-lying levels including the ground state [5-7,28,42,43]. Average branching ratios derived from the present cascade simulations are illustrated in Fig. 5. By dividing the summed intensities $N(E)$ in a bin of the experimental intensity distribution of the groundstate transitions with the corresponding branching ratio and transforming the intensities to a cross section analogous to Eq. (1), we obtain the absorption cross section in each bin as $\sigma_{\gamma}(E)=\sigma_{\gamma \gamma}(E) / b_{0}(E)$ for each nuclear realization. The uncertainty of the intensity in an energy bin was deduced as $\delta N(E)=\sqrt{N(E)}+\sum\left[\sqrt{N\left(E^{\prime}>E\right)} b\left(E^{\prime} \rightarrow E\right)\right]$, where $b\left(E^{\prime} \rightarrow E\right)$ is the branching intensity from bin $E^{\prime}$ to bin $E$. Finally, the absorption cross sections of each bin were obtained by averaging over the values of the nuclear realizations. The simulations were performed iteratively. The strength function obtained from an iteration step was used as the input for the next step. The iteration was stopped when the input strength function and the output strength function were in agreement within their uncertainties. The cross section obtained in the last (ninth) iteration step is taken as the final absorption cross section. The uncertainties of the cross-section values include statistical uncertainties of the spectrum, the given uncertainty of the efficiency, uncertainties of the flux resulting from the integrated cross sections of the ${ }^{11} \mathrm{~B}$ levels, and the mentioned uncertainties of the level-density parameters.

Toward low energy, the uncertainties increase due to the use of the spectrum-stripping method and the cross sections do not converge. Therefore, cross sections cannot be determined below an excitation energy of $7 \mathrm{MeV}$ in the high-energy measurement. To obtain values at lower energies, the same procedure was applied to the low-energy measurement.

\section{DISCUSSION}

The experimental photoabsorption cross sections resulting from the procedure just described for the $\left(\gamma, \gamma^{\prime}\right)$ data obtained from the measurements at $E_{e}=7.5$ and $13.9 \mathrm{MeV}$ are listed in Tables II and III and are graphed in Fig. 6 together with the experimental cross section for the $(\gamma, n)$ reaction [54]. The $\left(\gamma, \gamma^{\prime}\right)$ cross section is characterized by several peaks rather than by a smooth curve. Most of these peaks correspond to the prominent excitations seen in Table I and Figs. 1 and 4, which appear on top of the quasicontinuum. This structure resembles the ones found for other nuclides near closed shells, such as the $N=50$ isotones $[5,6,26,55]$, whereas the absorption cross sections of open-shell nuclides are rather smooth curves, for example, the ones in ${ }^{74} \mathrm{Ge}[39],{ }^{128} \mathrm{Xe}$ [12], and ${ }^{181} \mathrm{Ta}$ [42]. In addition to the experimental data, the TLO with the parameters just mentioned and the photoabsorption cross section given in the latest TALYS-based evaluated nuclear data library (TENDL-2019) [56] are displayed. For the latter, the strength of the GDR used as an input for the TALYS code [57] was scaled with a factor of 1.45 [56], which leads to large values compared to the $(\gamma, n)$ data and the TLO. The TENDL cross section is the sum of the ones of various reaction channels, which are shown in Fig. 7. The $\left(\gamma, \gamma^{\prime}\right)$ cross section is predicted with magnitudes comparable with the experimental ones, neglecting, however, the pronounced resonancelike structures found in the experiment. The $(\gamma, p)$ channel dominates the total cross section from about $12 \mathrm{MeV}$ up to about $25 \mathrm{MeV}$ in this $N=Z+2$ nuclide. The predicted $(\gamma, n)$ cross section underestimates the experimental one shown in Fig. 6 by a factor of about 2.5 at its maximum and is also considerably smaller than the predicted $(\gamma, p)$ cross section, which dominates the GDR in the region of its maximum.

The peaks in the experimental $\left(\gamma, \gamma^{\prime}\right)$ cross section are caused by prominent transitions from $1^{+}$as well as $1^{-}$states. This is similar to the characteristics of other nuclides in this mass region as mentioned in Sec. I, whereas the structures of nuclides around $N=50$ include exclusively strong excitations of $1^{-}$states. The relation between $E 1$ and $M 1$ strength in ${ }^{54} \mathrm{Fe}$ can be analyzed for the transitions from states with parity assignments given in Table I. The summed integrated 
TABLE II. Absorption cross section deduced from the present $\left(\gamma, \gamma^{\prime}\right)$ experiment at $E_{e}=7.5 \mathrm{MeV}$.

\begin{tabular}{ll}
\hline \hline$E_{\gamma}(\mathrm{MeV})$ & $\sigma(\mathrm{mb})^{\mathrm{a}}$ \\
\hline 4.3 & $0.16(4)$ \\
4.4 & $0.24(8)$ \\
4.5 & $0.017(9)$ \\
4.6 & $0.31(15)$ \\
4.9 & $0.50(5)$ \\
5.0 & $0.33(10)$ \\
5.1 & $0.49(8)$ \\
5.2 & $0.18(5)$ \\
5.3 & $0.15(3)$ \\
5.4 & $0.21(5)$ \\
5.5 & $0.18(8)$ \\
5.7 & $0.26(4)$ \\
5.8 & $0.12(6)$ \\
5.9 & $0.12(5)$ \\
6.0 & $0.41(18)$ \\
6.1 & $0.70(27)$ \\
6.2 & $0.25(3)$ \\
6.3 & $0.53(7)$ \\
6.4 & $0.45(8)$ \\
6.5 & $1.04(10)$ \\
6.6 & $0.45(4)$ \\
6.7 & $0.60(5)$ \\
6.8 & $0.82(5)$ \\
6.9 & $0.63(8)$ \\
7.0 & $0.44(5)$ \\
7.1 & $0.72(5)$ \\
7.2 & $0.49(4)$ \\
7.3 & $0.75(5)$ \\
7.4 & $1.58(8)$ \\
7.5 & $1.19(7)$ \\
\hline \hline
\end{tabular}

a Absorption cross section resulting from the experimental intensity distribution including the quasicontinuum, corrected for branching intensities and branching ratios obtained from $\gamma$-ray cascade simulations. The uncertainties include statistical uncertainties of the included intensities as described in Sec. III, a 5\% uncertainty of the detector efficiency, and uncertainties of the photon flux based on the uncertainties of the cross sections in ${ }^{11} \mathrm{~B}$. Systematic uncertainties of strength-function and level-density models can cause additional uncertainties of up to about $20 \%$ not included here.

scattering cross section of all definite and tentative $1^{-}$states in Table I amounts to $\sum I_{s}\left(1^{-}\right)=2394(65) \mathrm{eV}$ b and that of all $1^{+}$states amounts to $\sum I_{s}\left(1^{+}\right)=1999(53) \mathrm{eV}$ b. The contribution of $M 1$ strength to the total strength is hence about $45 \%$ and that of $E 1$ strength is about $55 \%$. Reduced transition strengths $B(E 1)$ and $B(M 1)$ were deduced assuming branching ratios $b_{0}=1$ for the ground-state transitions, because transitions from $J=1$ states to the $2_{1}^{+}$state, which are expected to be the most intense branching transitions other than the ground-state transitions, were not clearly identified in the respective spectra measured at $\mathrm{HI} \gamma \mathrm{S}$. The intensities of the weakly visible $2_{1}^{+} \rightarrow 0_{1}^{+}$transition have not been used to estimate branching ratios because the $2_{1}^{+}$state may also collect feeding intensities from other states simultaneously excited with the considered one by a given photon beam of up
TABLE III. Absorption cross section deduced from the present $\left(\gamma, \gamma^{\prime}\right)$ experiment at $E_{e}=13.9 \mathrm{MeV}$.

\begin{tabular}{|c|c|}
\hline$E_{\gamma}(\mathrm{MeV})$ & $\sigma(\mathrm{mb})^{\mathrm{a}}$ \\
\hline 7.0 & $1.1(8)$ \\
\hline 7.2 & $3.3(31)$ \\
\hline 7.3 & $1.6(8)$ \\
\hline 7.4 & $1.2(8)$ \\
\hline 7.5 & 2.1(13) \\
\hline 7.7 & 2.6(19) \\
\hline 7.8 & $3.6(14)$ \\
\hline 8.1 & $5.8(41)$ \\
\hline 8.2 & $12.2(22)$ \\
\hline 8.3 & $2.9(8)$ \\
\hline 8.4 & $3.3(7)$ \\
\hline 8.5 & $3.7(8)$ \\
\hline 8.6 & $6.7(17)$ \\
\hline 8.7 & $3.6(9)$ \\
\hline 8.8 & $3.6(11)$ \\
\hline 8.9 & 4.2(13) \\
\hline 9.0 & $14.8(34)$ \\
\hline 9.1 & 8.1(16) \\
\hline 9.2 & $7.8(13)$ \\
\hline 9.3 & $4.6(8)$ \\
\hline 9.4 & $11.0(25)$ \\
\hline 9.5 & 4.1(13) \\
\hline 9.6 & $6.7(10)$ \\
\hline 9.7 & $4.5(13)$ \\
\hline 9.8 & $5.9(8)$ \\
\hline 9.9 & $8.4(10)$ \\
\hline 10.0 & $10.9(9)$ \\
\hline 10.1 & $17.5(14)$ \\
\hline 10.2 & $14.4(11)$ \\
\hline 10.3 & $11.6(9)$ \\
\hline 10.4 & $8.9(7)$ \\
\hline 10.5 & $18.0(13)$ \\
\hline 10.6 & $6.6(6)$ \\
\hline 10.7 & $7.6(6)$ \\
\hline 10.8 & $7.9(6)$ \\
\hline 10.9 & $9.5(9)$ \\
\hline 11.0 & $10.2(8)$ \\
\hline 11.1 & $10.7(8)$ \\
\hline 11.2 & $12.4(7)$ \\
\hline 11.3 & $12.6(6)$ \\
\hline 11.4 & $7.6(5)$ \\
\hline 11.5 & $14.0(11)$ \\
\hline 11.6 & $8.5(7)$ \\
\hline 11.7 & $6.8(7)$ \\
\hline 11.8 & $9.4(9)$ \\
\hline 11.9 & $9.5(9)$ \\
\hline 12.0 & $8.4(9)$ \\
\hline 12.1 & 7.7(9) \\
\hline 12.2 & $7.3(8)$ \\
\hline 12.3 & $9.7(11)$ \\
\hline 12.4 & $8.9(10)$ \\
\hline 12.5 & 7.4(10) \\
\hline 12.6 & $8.0(10)$ \\
\hline 12.7 & $9.3(12)$ \\
\hline 12.8 & $6.1(9)$ \\
\hline 12.9 & $10.7(13)$ \\
\hline 13.0 & 7.3(11) \\
\hline
\end{tabular}


TABLE III. (Continued.)

\begin{tabular}{ll}
\hline$E_{\gamma}(\mathrm{MeV})$ & $\sigma(\mathrm{mb})^{\mathrm{a}}$ \\
\hline 13.1 & $5.4(9)$ \\
13.2 & $9.8(13)$ \\
13.3 & $6.2(11)$ \\
13.4 & $5.5(9)$ \\
\hline \hline
\end{tabular}

a Absorption cross section resulting from the experimental intensity distribution including the quasicontinuum, corrected for branching intensities and branching ratios obtained from $\gamma$-ray cascade simulations. The uncertainties include statistical uncertainties of the included intensities as described in Sec. III, a 5\% uncertainty of the detector efficiency, and uncertainties of the photon flux based on the uncertainties of the cross sections in ${ }^{11} \mathrm{~B}$. Systematic uncertainties of strength-function and level-density models can cause additional uncertainties of up to about $20 \%$ not included here.

to about $300 \mathrm{keV}$ energy spread at the highest energies. The $B(E 1)$ and $B(M 1)$ values determined in this way are given in Tables IV and V, respectively. The summed strengths result in $\sum B(E 1)=0.0215(6) e^{2} \mathrm{fm}^{2}$ and $\sum B(M 1)=1.59(4) \mu_{N}^{2}$. Note that these summed strengths are not the full strengths because the transitions without parity assignments in Table I are not included. Furthermore, unobserved transitions in the quasicontinuum contribute to the summed strengths as well. Nevertheless, these summed strengths are of magnitudes similar to the ones found for ${ }^{50} \mathrm{Cr}$ [13] and for the $N=28$ isotone ${ }^{52} \mathrm{Cr}[15,16]$. The distribution of $M 1$ strength is compared with predictions of shell-model calculations in the following.

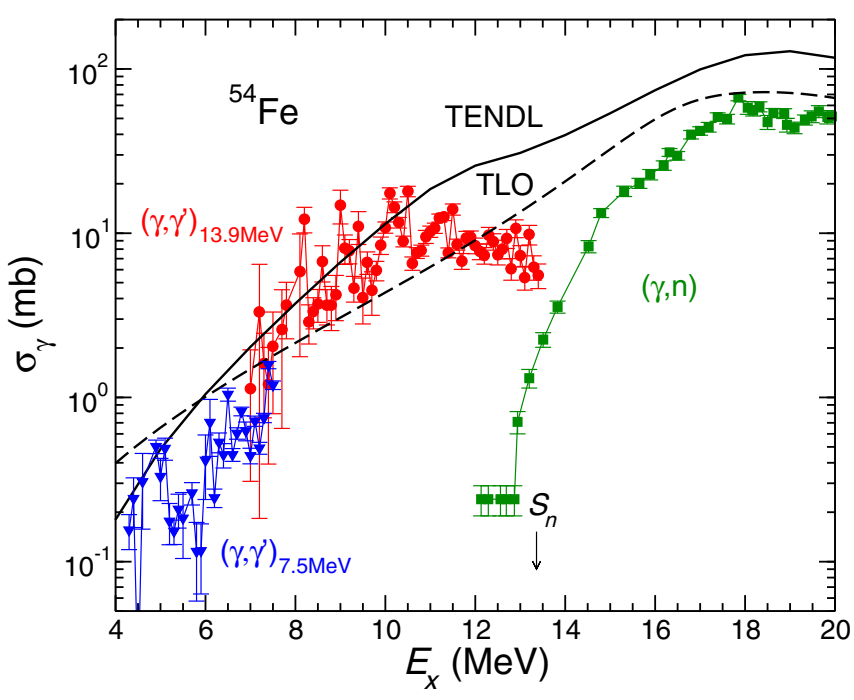

FIG. 6. Photoabsorption cross sections of ${ }^{54} \mathrm{Fe}$ resulting from the present $\left(\gamma, \gamma^{\prime}\right)$ experiments at $E_{e}=7.5 \mathrm{MeV}$ (blue triangles) and $E_{e}=13.9 \mathrm{MeV}$ (red circles), from $(\gamma, n)$ data taken from Ref. [54] (green squares), from calculations using the TALYS code as given in the TENDL-2019 library (black solid line), and from the TLO with parameters given in the text (black dashed line).

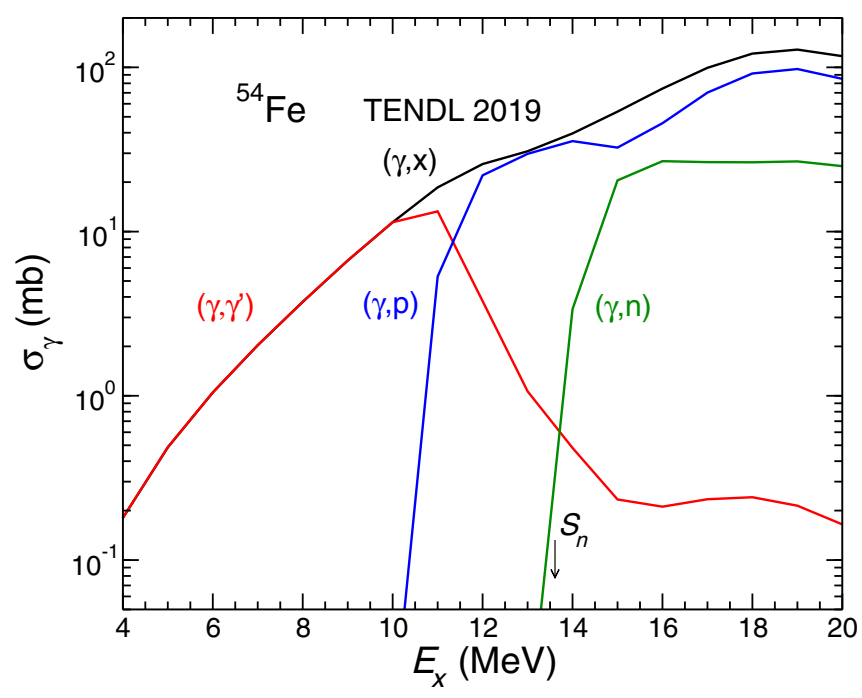

FIG. 7. Photoabsorption cross section of ${ }^{54} \mathrm{Fe}$ (black line) together with the partial cross sections for the reaction channels $\left(\gamma, \gamma^{\prime}\right)$ (red line), $(\gamma, n)$ (green line), and $(\gamma, p)$ (blue line) taken from the TENDL-2019 library.

\section{SHELL-MODEL CALCULATIONS}

Shell-model calculations for ${ }^{54} \mathrm{Fe}$ were carried out with the GX1A Hamiltonian [58,59] for the $f p$ shell using the code NUSHELLX@MSU [60]. The model space included the proton and neutron orbits $\left(0 f_{7 / 2}, 1 p_{3 / 2}, 0 f_{5 / 2}, 1 p_{1 / 2}\right)$ without limits in occupation numbers. The calculations were performed for the lowest 120 states with $J^{\pi}=1^{+}$. Reduced transition strengths $B\left(M 1,1^{+} \rightarrow 0_{1}^{+}\right)$were calculated using effective $g$ factors of $g_{s}^{\text {eff }}=0.74 g_{s}^{\text {free }}$ [61]. The experimental $B\left(M 1,1^{+} \rightarrow 0_{1}^{+}\right)$values given in Table $\mathrm{V}$ are compared with calculated ones in Fig. 8. Out of the 120 calculated values, only the 53 values greater than $0.005 \mu_{N}^{2}$ are shown. This lower limit corresponds roughly to the smallest experimental values. The first calculated $1^{+}$state appears at $4.560 \mathrm{MeV}$, whereas the first experimental state with a $1^{+}$assignment is the one at $6.841 \mathrm{MeV}$ (cf. Table I). However, one or more of the states between 5.0 and $5.5 \mathrm{MeV}$, which were not covered by the experiments at $\mathrm{HI} \gamma \mathrm{S}$, may also have spin and parity $1^{+}$. The running sums of the $B(M 1)$ values are graphed in Fig. 9. Both the experimental and calculated curves show a steplike behavior caused by strong peaks in the $B(M 1)$ distributions. The steps of the calculated distribution appear by about $0.5 \mathrm{MeV}$ lower in energy than the experimental ones. The summed strength $\sum B(M 1)=1.76 \mu_{N}^{2}$ of the calculated values greater than $0.005 \mu_{N}^{2}$ between $E_{\gamma}=6.8$ and $10.6 \mathrm{MeV}$ exceeds the upper limit of the experimental value of $\sum B(M 1)=1.59(4) \mu_{N}^{2}$ by about $8 \%$. This relation resembles the one found for the isotone ${ }^{50} \mathrm{Cr}$ [16]. Possible reasons for the difference are that some of the states with unknown parity in Table I may be $1^{+}$states that are not taken into account in this comparison or may be unobserved branching transitions. 
TABLE IV. Experimental reduced $E 1$ transition strengths.

\begin{tabular}{lc}
\hline \hline$E_{\gamma}(\mathrm{MeV})$ & $B\left(E 1,1^{-} \rightarrow 0_{1}^{+}\right)\left(10^{-4} e^{2} \mathrm{fm}^{2}\right)^{\mathrm{a}}$ \\
\hline 6.128 & $2.03(20)$ \\
6.523 & $1.28(17)$ \\
7.121 & $2.4(3)$ \\
8.015 & $35.6(3)$ \\
8.093 & $10.8(10)$ \\
8.220 & $59(5)$ \\
8.354 & $3.5(4)$ \\
8.450 & $2.25(29)$ \\
8.897 & $6.2(6)$ \\
9.342 & $2.7(4)$ \\
9.515 & $4.3(6)$ \\
10.101 & $4.3(5)$ \\
10.240 & $4.7(6)$ \\
10.250 & $3.4(5)$ \\
10.339 & $11.1(10)$ \\
10.377 & $2.6(7)$ \\
10.488 & $1.7(6)$ \\
10.510 & $5.6(9)$ \\
10.591 & $1.6(5)$ \\
10.701 & $3.6(4)$ \\
10.880 & $2.2(3)$ \\
10.891 & $2.0(3)$ \\
10.926 & $1.14(23)$ \\
11.022 & $7.9(13)$ \\
11.194 & $7.3(7)$ \\
11.211 & $2.7(3)$ \\
11.314 & $3.4(4)$ \\
11.334 & $5.4(6)$ \\
11.531 & $2.7(4)$ \\
\hline & $11.2(11)$ \\
\hline & \\
\hline &
\end{tabular}

a Value obtained from the $I_{s}$ of the corresponding state given in Table I, using the relation $B(E 1)=0.000955\left(\Gamma_{0} / \mathrm{meV}\right) /\left(E_{\gamma} / \mathrm{meV}\right)^{3}$ and Eq. (2) with $\Gamma_{0} / \Gamma=1$. The uncertainty of the $B(E 1)$ value was percentagewise deduced from that of $I_{s}$.

To get an impression about the fraction of orbital and spin contributions to the $B(M 1)$ values, calculations with $g_{s}^{\pi}=$ 0.001 and $g_{s}^{v}=-0.001$ were carried out. The ratios of the values with and without spin contribution scatter over 8 orders of magnitude for the individual transitions, reaching from almost exclusive spin contributions over dominating orbital contributions $\left[B(M 1) / B(M 1)_{g_{s}=0} \approx 1\right]$ to values smaller than 1 in cases of destructive superposition of orbital and spin parts. There is no clear tendency of these values with varying energy.

In earlier shell-model studies of ${ }^{54} \mathrm{Fe}$ [62] and of the neighboring isotope ${ }^{56} \mathrm{Fe}[63]$ using the code NUSHELLX, a truncated model space was applied to make the calculations feasible for a greater spin range and hence a much bigger number of transitions. The lowest 40 states with $J^{\pi}=2^{+}$ calculated with a truncated model space including the proton orbits $\left(0 f_{7 / 2}\right)^{6-l}\left(1 p_{3 / 2}, 0 f_{5 / 2}, 1 p_{1 / 2}\right)^{l}$, with $l=0,1$, and 2 , and the neutron orbits $\left(0 f_{7 / 2}\right)^{8-m}\left(1 p_{3 / 2}, 0 f_{5 / 2}, 1 p_{1 / 2}\right)^{m}$, with $m=0,1$, and 2 , are compared with the ones obtained from the present calculations including the full $f p$ shell for protons
TABLE V. Experimental reduced $M 1$ transition strengths.

\begin{tabular}{lc}
\hline \hline$E_{\gamma}(\mathrm{MeV})$ & $B\left(M 1,1^{+} \rightarrow 0_{1}^{+}\right)\left(10^{-2} \mu_{N}^{2}\right)^{\mathrm{a}}$ \\
\hline 6.841 & $0.71(19)$ \\
6.914 & $0.68(14)$ \\
8.119 & $13.7(12)$ \\
8.338 & $5.5(5)$ \\
8.618 & $2.6(3)$ \\
8.856 & $8.6(7)$ \\
8.986 & $11.9(11)$ \\
9.065 & $10.8(10)$ \\
9.113 & $7.7(7)$ \\
9.149 & $8.5(7)$ \\
9.307 & $6.4(6)$ \\
9.410 & $14.2(13)$ \\
9.538 & $3.7(6)$ \\
9.909 & $2.9(5)$ \\
9.925 & $2.9(4)$ \\
10.060 & $16.7(15)$ \\
10.141 & $9.5(9)$ \\
10.186 & $8.6(10)$ \\
10.538 & $23.3(21)$ \\
\hline \hline
\end{tabular}

a Value deduced from the $I_{s}$ of the corresponding state given in Table I, using the relation $B(M 1)=0.0866\left(\Gamma_{0} / \mathrm{meV}\right) /\left(E_{\gamma} / \mathrm{meV}\right)^{3}$ and Eq. (2) with $\Gamma_{0} / \Gamma=1$. The uncertainty of the $B(M 1)$ value was percentagewise deduced from that of $I_{s}$.

and neutrons in Fig. 10. The states are lower in energy in the full configuration space than those in the truncated space. For example, the energy of the $2_{1}^{+}$state changes from $1.467 \mathrm{MeV}$ in the limited space to $1.449 \mathrm{MeV}$ in the full space and the $B\left(E 2,2_{1}^{+} \rightarrow 0_{1}^{+}\right)$value changes from 108 to $149 e^{2} \mathrm{fm}^{4}$, whereas the experimental values are $E\left(2_{1}^{+}\right)=1.408 \mathrm{MeV}$ and $B\left(E 2,2_{1}^{+} \rightarrow 0_{1}^{+}\right)=135(4) e^{2} \mathrm{fm}^{4}$ [34]. In the considered energy range up to $10.6 \mathrm{MeV}$, there are $401^{+}$states calculated

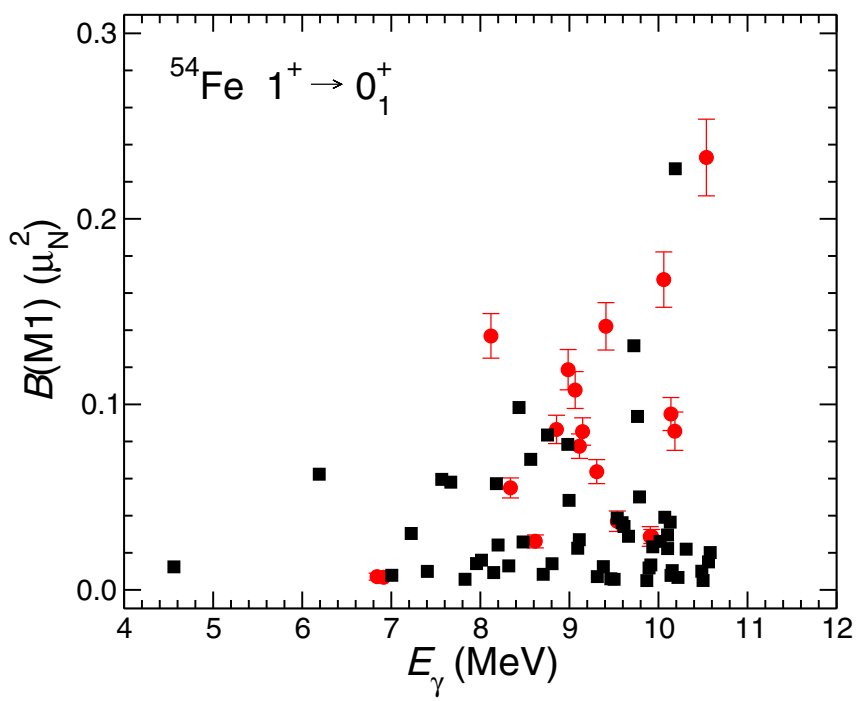

FIG. 8. Experimental $B\left(M 1,1^{+} \rightarrow 0_{1}^{+}\right)$values (red circles) and calculated values $B\left(M 1,1^{+} \rightarrow 0_{1}^{+}\right)>0.005 \mu_{N}^{2}$ from the lowest 120 $1^{+}$states (black squares). 


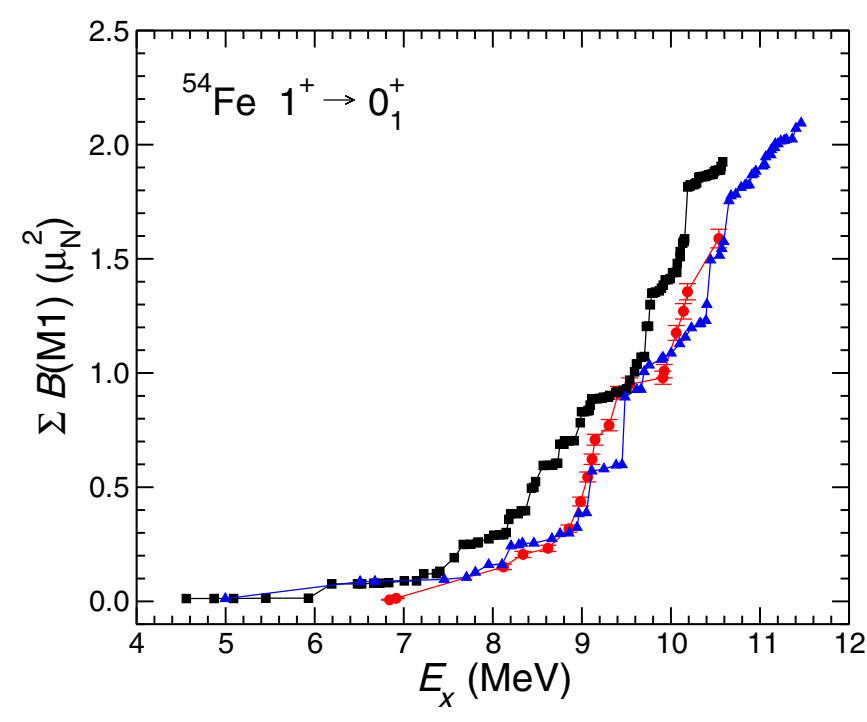

FIG. 9. Running sums $\sum B\left(M 1,1^{+} \rightarrow 0_{1}^{+}\right.$) of experimental (red circles) and calculated values (black squares). Also shown are the values obtained in a truncated model space (blue triangles) discussed in the text.

in the limited space, but 120 in the full space, which are compared in Fig. 11. This shows that the $M 1$ strength is shifted down by almost $1 \mathrm{MeV}$ in the full model space compared to the truncated model space. The running sum $\sum B\left(M 1,1^{+} \rightarrow 0_{1}^{+}\right)$including the lowest 65 states obtained with the limited space is also shown in Fig. 9. The lowering of states in the full space compared with the ones in the truncated space is reflected by a shift of the steps toward low energy. The curves shown in Fig. 9 suggest a better agreement of the experimental running sum with the one in the truncated space compared to the one in the full space. One must, however, be aware that the experimental strengths are not complete and

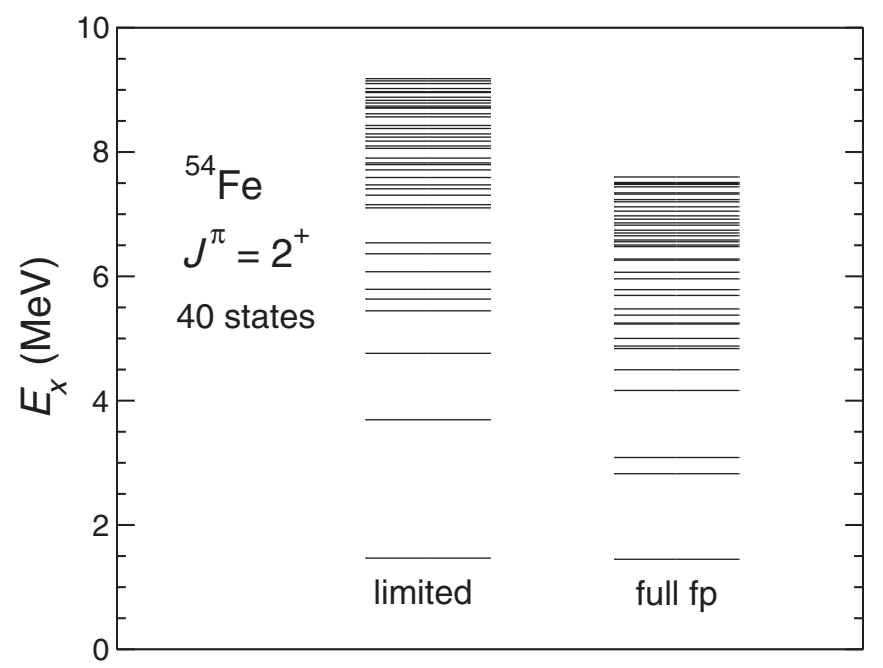

FIG. 10. Energies of the lowest 40 states with $J^{\pi}=2^{+}$calculated in a model space with limited occupation numbers and in the full $f p$ space.

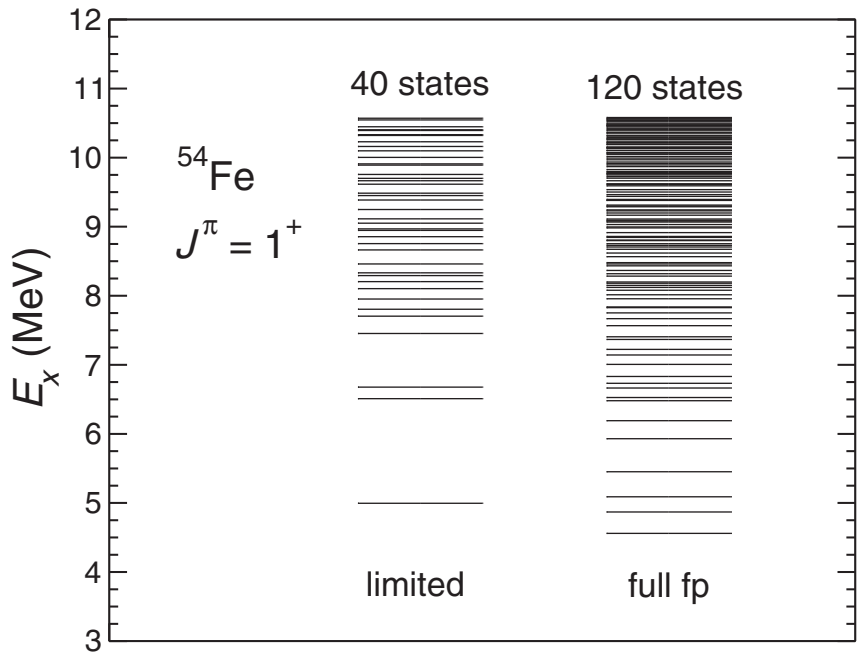

FIG. 11. Energies of the lowest 40 states with $J^{\pi}=1^{+}$up to $10.6 \mathrm{MeV}$ calculated in a model space with limited occupation numbers and the 120 states up to $10.6 \mathrm{MeV}$ in the full $f p$ space.

the strengths from the truncated space are less reliable than the ones from the full space.

As an alternative description, the $B(M 1)$ distribution in ${ }^{54} \mathrm{Fe}$ was also computed in the full $f p$ shell with the LenziNowacki-Poves-Sieja interaction [64] using the code ANTOINE [65], employing the Lanczos strength-function method with 300 iterations. A quenching factor of 0.75 was applied on the spin part of the magnetic operator. This calculation predicts the first $1^{+}$state at $5.0 \mathrm{MeV}$ with a $B\left(M 1,1^{+} \rightarrow 0_{1}^{+}\right)$value of $0.014 \mu_{N}^{2}$. There are 31 states located below $10.6 \mathrm{MeV}$ and the summed strength from 6.8 to $10.6 \mathrm{MeV}$ amounts to $\sum B(M 1)=2.05 \mu_{N}^{2}$, being even larger than that from the GX1A shell-model calculation and thus exceeding the experimental value by about $30 \%$.

\section{SUMMARY}

The dipole-strength distribution in ${ }^{54} \mathrm{Fe}$ up to the neutronseparation energy was studied in photon-scattering experiments at the $\gamma$ ELBE facility using bremsstrahlung at two different electron energies and at the $\mathrm{HI} \gamma \mathrm{S}$ facility using quasimonoenergetic photon beams of 26 photon energies. About 100 levels were identified. Spins $J=1$ were deduced from angular correlations of ground-state transitions measured at $\gamma$ ELBE and the parities of states were determined from azimuthal asymmetries of intensities measured at $\mathrm{HI} \gamma \mathrm{S}$. The intensity distribution obtained from the measured spectra after a correction for detector response and a subtraction of atomic background in the target contains a continuum part in addition to the resolved peaks, which was included in the determination of the photoabsorption cross section. An assignment of inelastic transitions to particular levels, and thus the determination of branching ratios, was in general not possible. To get information about the intensities of inelastic transitions to low-lying levels we have applied statistical methods. By means of simulations of $\gamma$-ray cascades, 
intensities of branching transitions were estimated and subtracted from the experimental intensity distribution and the intensities of ground-state transitions could be corrected in average for their branching ratios. In this way, a continuous photoabsorption cross section was derived for the energy range from about $4 \mathrm{MeV}$ up to the neutron threshold at 13.3 $\mathrm{MeV}$, which can be combined with the $(\gamma, p)$ and $(\gamma, n)$ cross sections toward higher energies. The data show that the $M 1$ strength amounts to about $45 \%$ and the $E 1$ strength to about $55 \%$ of the strength in the resolved transitions, which compares to the findings for neighboring nuclides. This contribution of $M 1$ strength is considerably higher than in heavier nuclides, which are dominated by up to more than $90 \%$ of $E 1$ strength in the considered energy region. Consequently, the phenomenological parametrizations of $M 1$ strength as used in statistical reaction-model calculations may be reconsidered for nuclides in the mass-60 region. The experimental $B(M 1)$ values were compared with predictions of shell-model calcu- lations. Most of the calculated $B\left(M 1,1^{+} \rightarrow 0_{1}^{+}\right)$values are dominated by spin contributions.

\section{ACKNOWLEDGMENTS}

We thank the operating crews of the ELBE accelerator and of the TUNL storage ring and FEL for their cooperation and we thank K. Römer for the technical assistance during the experiments. This work was partially supported by the US Department of Energy (DOE), Office of Nuclear Physics, under Grant No. DE-FG02-97ER41033. B.A.B. acknowledges support by the NSF under Grant No. PHY-1811855 and R.M. acknowledges support by the US Department of Energy, Office of Science, Office of Nuclear Physics, under Grant No. LANLE9BW. R.S. gratefully acknowledges the allocation of computing time through the Centers for HighPerformance Computing of Technische Universität Dresden and of Helmholtz-Zentrum Dresden-Rossendorf.
[1] T. Kawano et al., Nucl. Data Sheets 163, 109 (2020).

[2] S. Goriely et al., Eur. Phys. J. A 55, 172 (2019).

[3] R. Schwengner et al., Nucl. Instrum. Methods Phys. Res., Sect. A 555, 211 (2005).

[4] H. R. Weller, M. W. Ahmed, H. Gao, W. Tornow, Y. K. Wu, M. Gai, and R. Miskimen, Prog. Part. Nucl. Phys. 62, 257 (2009).

[5] R. Schwengner et al., Phys. Rev. C 87, 024306 (2013).

[6] G. Rusev et al., Phys. Rev. C 79, 061302(R) (2009).

[7] R. Massarczyk et al., Phys. Rev. Lett. 112, 072501 (2014).

[8] G. A. Bartholomew, E. D. Earle, A. J. Ferguson, J. W. Knowles, and M. A. Lone, Adv. Nucl. Phys. 7, 229 (1973).

[9] D. Savran, T. Aumann, and A. Zilges, Prog. Part. Nucl. Phys. 70, 210 (2013).

[10] A. Bracco, E. G. Lanza, and A. Tamii, Prog. Part. Nucl. Phys. 106, 360 (2019).

[11] R. Raut et al., Phys. Rev. Lett. 111, 112501 (2013).

[12] R. Massarczyk, G. Rusev, R. Schwengner, F. Dönau, C. Bhatia, M. E. Gooden, J. H. Kelley, A. P. Tonchev, and W. Tornow, Phys. Rev. C 90, 054310 (2014).

[13] H. Pai et al., Phys. Rev. C 93, 014318 (2016).

[14] P. C. Ries et al., Phys. Rev. C 100, 021301(R) (2019).

[15] T. Shizuma, T. Hayakawa, I. Daito, H. Ohgaki, S. Miyamoto, and F. Minato, Phys. Rev. C 96, 044316 (2017).

[16] J. Wilhelmy et al., Phys. Rev. C 98, 034315 (2018).

[17] T. Shizuma, T. Hayakawa, H. Ohgaki, H. Toyokawa, T. Komatsubara, N. Kikuzawa, T. Inakura, M. Honma, and H. Nakada, Phys. Rev. C 87, 024301 (2013).

[18] M. Scheck et al., Phys. Rev. C 87, 051304(R) (2013).

[19] H. Lancman, R. J. Sparks, and C. Van Der Leun, Nucl. Phys. A 257, 29 (1976).

[20] F. Ajzenberg-Selove, Nucl. Phys. A 506, 1 (1990).

[21] G. Rusev, A. P. Tonchev, R. Schwengner, C. Sun, W. Tornow, and Y. K. Wu, Phys. Rev. C 79, 047601 (2009).

[22] S. Agostinelli et al., Nucl. Instrum. Methods Phys. Res., Sect. A 506, 250 (2003).

[23] J. Allison et al., IEEE Trans. Nucl. Sci. 53, 270 (2006).

[24] J. Allison et al., Nucl. Instrum. Methods Phys. Res., Sect. A 835, 186 (2016).
[25] G. Rusev, Dissertation, Technische Universität, Dresden, 2007, Report FZD-478, http://www.hzdr.de/publications/010008/ 10008.pdf.

[26] R. Schwengner et al., Phys. Rev. C 76, 034321 (2007).

[27] G. Rusev et al., Phys. Rev. C 77, 064321 (2008).

[28] R. Massarczyk et al., Phys. Rev. C 86, 014319 (2012).

[29] E. Trompler, Diploma thesis, Technische Universität, Dresden, 2009, Report FZD-523, http://www.hzdr.de/publications/ 013364/13364.pdf.

[30] S. Carson, C. Iliadis, J. Cesaratto, A. Champagne, L. Downen, M. Ivanovic, J. Kelley, R. Longland, J. R. Newton, G. Rusev, and A. P. Tonchev, Nucl. Instrum. Methods Phys. Res., Sect. A 618, 190 (2010).

[31] E. Haug, Radiat. Phys. Chem. 77, 207 (2008).

[32] G. Roche, C. Ducos, and J. Proriol, Phys. Rev. A 5, 2403 (1972)

[33] F. Salvat, J. D. Martinez, R. Mayol, and J. Parellada, Phys. Rev. A 36, 467 (1987).

[34] Y. Dong and H. Junde, Nucl. Data Sheets 121, 1 (2014).

[35] R. Jenkins, R. W. Gould, and D. Gedke, Quantitative X-Ray Spectroscopy (Dekker, New York, 1995).

[36] E. Kwan et al., Phys. Rev. C 83, 041601(R) (2011).

[37] N. Pietralla, Z. Berant, V. N. Litvinenko, S. Hartman, F. F. Mikhailov, I. V. Pinayev, G. Swift, M. W. Ahmed, J. H. Kelley, S. O. Nelson, R. Prior, K. Sabourov, A. P. Tonchev, and H. R. Weller, Phys. Rev. Lett. 88, 012502 (2001).

[38] A. K. Furr, E. L. Robinson, and C. H. Robins, Nucl. Instrum. Methods 63, 205 (1968).

[39] R. Massarczyk et al., Phys. Rev. C 92, 044309 (2015).

[40] A. Makinaga et al., Phys. Rev. C 94, 044304 (2016).

[41] T. Shizuma et al., Phys. Rev. C 98, 064317 (2018).

[42] A. Makinaga et al., Phys. Rev. C 90, 044301 (2014).

[43] G. Schramm et al., Phys. Rev. C 85, 014311 (2012).

[44] F. Bečvář, Nucl. Instrum. Methods Phys. Res., Sect. A 417, 434 (1998).

[45] C. E. Porter and R. G. Thomas, Phys. Rev. 104, 483 (1956).

[46] A. Gilbert and A. G. W. Cameron, Can. J. Phys. 43, 1446 (1965). 
[47] T. von Egidy and D. Bucurescu, Phys. Rev. C 80, 054310 (2009).

[48] S. I. Al-Quraishi, S. M. Grimes, T. N. Massey, and D. A. Resler, Phys. Rev. C 67, 015803 (2003).

[49] A. R. Junghans, G. Rusev, R. Schwengner, A. Wagner, and E. Grosse, Phys. Lett. B 670, 200 (2008).

[50] E. Grosse, A. R. Junghans, and R. Massarczyk, Eur. Phys. J. A 53, 225 (2017).

[51] S. Raman, C. W. Nestor, Jr., and P. Tikkanen, At. Data Nucl. Data Tables 78, 1 (2001).

[52] J.-P. Delaroche, M. Girod, J. Libert, H. Goutte, S. Hilaire, S. Péru, N. Pillet, and G. F. Bertsch, Phys. Rev. C 81, 014303 (2010).

[53] R. Capote et al., Nucl. Data Sheets 110, 3107 (2009).

[54] J. W. Norbury, M. N. Thompson, K. Shoda, and H. Tsubota, Aust. J. Phys. 31, 471 (1978).

[55] R. Schwengner et al., Phys. Rev. C 78, 064314 (2008).
[56] A. J. Koning, D. Rochman, J. Sublet, N. Dzysiuk, M. Fleming, and S. van der Marck, Nucl. Data Sheets 155, 1 (2019).

[57] A. J. Koning and D. Rochman, Nucl. Data Sheets 113, 2841 (2012), and references therein.

[58] M. Honma, T. Otsuka, B. A. Brown, and T. Mizusaki, Phys. Rev. C 69, 034335 (2004).

[59] M. Honma, T. Otsuka, B. A. Brown, and T. Mizusaki, Eur. Phys. J. A 25, 499 (2005).

[60] B. A. Brown and W. D. M. Rae, Nucl. Data Sheets 120, 115 (2014).

[61] M. Honma, T. Otsuka, T. Mizusaki, M. Hjorth-Jensen, and B. A. Brown, J. Phys.: Conf. Ser. 20, 7 (2005).

[62] R. Schwengner and G. Rusev, Phys. Rev. C 100, 054320 (2019).

[63] B. A. Brown and A. C. Larsen, Phys. Rev. Lett. 113, 252502 (2014).

[64] S. M. Lenzi, F. Nowacki, A. Poves, and K. Sieja, Phys. Rev. C 82, 054301 (2010).

[65] E. Caurier and F. Nowacki, Acta Phys. Pol., B 30, 705 (1999). 